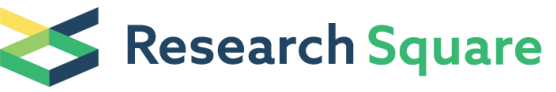 \\ Preprints are preliminary reports that have not undergone peer review. They should not be considered conclusive, used to inform clinical practice, or referenced by the media as validated information.
}

\section{Accelerated Brain Aging in Mild Traumatic Brain Injury: Longitudinal Pattern Recognition With White Matter Integrity}

\section{Shuoqiu Gan}

The Key Laboratory of Biomedical Information Engineering, Ministry of Education, Department of Biomedical Engineering, School of Life Science and Technology, Xi'an Jiaotong University; Department of Medical Imaging, the First Affiliated Hospital of Xi'an Jiaotong University

\section{Wen Shi}

The key Laboratory of Biomedical Information Engineering, Ministry of Education, Department of Biomedical Engineering, School of Life Science and Technology, Xi'an Jiaotong University; Institute for Medical Imaging Technology, School of Biomedical Engineering, Shanghai Jiao Tong University

\section{Shan Wang}

The Key Laboratory of Biomedical Information Engineering, Ministry of Education, Department of Biomedical Engineering, School of Life Science and Technology, Xi'an Jiaotong University

\section{Yingxiang Sun}

Department of Medical Imaging, the First Affiliated Hospital of Xi'an Jiaotong University

Bo Yin

Department of Neurosurgery, The Second Affiliated Hospital and Yuying Children's Hospital of Wenzhou Medical University

\section{Guanghui Bai}

Department of Radiology, the Second Affiliated Hospital and Yuying Children's Hospital of Wenzhou Medical University

\section{Xiaoyan Jia}

The Key Laboratory of Biomedical Information Engineering, Ministry of Education, Department of Biomedical Engineering, School of Life Science and Technology, Xi'an Jiaotong University

\section{Chuanzhu Sun}

The Key Laboratory of Biomedical Information Engineering, Ministry of Education, Department of Biomedical Engineering, School of Life Science and Technology, Xi'an Jiaotong University

\section{Xuan Niu}

Department of Medical Imaging, the First Affiliated Hospital of Xi'an Jiaotong University

\section{Zhuonan Wang}

Department of Medical Imaging, the First Affiliated Hospital of Xi'an Jiaotong University

\section{Xiaofan Jiang}

Department of Neurosurgery, Xijing Hospital, Fourth Military Medical University 


\section{Jun Liu}

Department of Radiology, The Second Xiangya Hospital, Central South University

\section{Ming Zhang}

Department of Medical Imaging, the First Affiliated Hospital of Xi'an Jiaotong University

\section{Lijun Bai ( $\sim$ bailijun@xjtu.edu.cn )}

Xi'an Jiaotong University https://orcid.org/0000-0001-8790-5424

\section{Research}

Keywords: mild traumatic brain injury, predicted age difference, accelerated brain-aging, relevance vector regression, white matter integrity

Posted Date: August 6th, 2020

DOI: https://doi.org/10.21203/rs.3.rs-54057/v1

License: (c) (i) This work is licensed under a Creative Commons Attribution 4.0 International License. Read Full License

Version of Record: A version of this preprint was published at Journal of Neurotrauma on May 19th, 2021. See the published version at https://doi.org/10.1089/neu.2020.7551. 


\section{Abstract}

Background: Long-term effects of mild traumatic brain injury (mTBI) resemble brain aging changes (i.e., microstructure integrity loss), which implies an accelerated age-associated process. This study aimed to develop a quantifiable neuroimaging marker to characterize the brain-aging process accelerated by $\mathrm{mTBI}$ from acute to chronic phases.

Methods: A brain-age prediction model was defined using relevance vector regression (RVR) in 523 healthy individuals, based on fractional anisotropy metrics from diffusion-tensor imaging. The model was adopted to estimate brain-predicted age difference (brain-PAD = predicted brain age - chronological age) in 116 acute mTBI patients and 63 healthy controls (HCs). Fifty patients were followed up 6 12 month post-injury to evaluate the longitudinal changes in brain-PAD. Another mTBI group containing 70 acute patients were included as a replicated cohort. We investigated whether brain-PAD was greater in patients with elderly age, post-concussion complaints, and risky apolipoprotein E (APOE) genotype, and whether it had the potential to predict neuropsychological outcomes for information processing speed (IPS). Between-group and longitudinal comparison in brain-PAD was conducted with analysis of covariance and linear mixed-effects model, respectively. The correlation between brain-PAD and continuous variables was analyzed with Spearman rank-order correlation.

Results: The RVR brain-age prediction model predicted brain age accurately $\left(r=0.96, R^{2}=0.93\right)$. The brain age of mTBI patients was estimated to be "older" in the acute phase, with mean brain-PAD of 2.59 ( \pm 5.97$)$ years compared with HCs $(0.12 \pm 3.19$ years $)(P<0.05)$ and replicated in another mTBI cohort (brain-PAD: $3.26 \pm 4.55$ years). The increased brain age in mTBI kept stable at 6-12 month post-injury (2.50 \pm 4.54 years). Patients with older age or severer post-concussion complaints obtained greater brain$\operatorname{PAD}(P<0.001, P=0.024)$, while patients with APOE $\varepsilon 4$ didn't obtain greater brain-PAD than those without. Additionally, brain-PAD in the acute phase predicted patients' IPS profile at $6 \sim 12$ month followup $(r h o=-0.36, P=0.01)$.

Conclusion: Mild TBI, even a single one, accelerates the brain-aging process. The brain-PAD can be considered as a quantitative neuroimaging marker to evaluate the susceptibility to neurodegeneration or other age-associated conditions following mTBI.

Trial registration: NCT02868684.

\section{Introduction}

Mild traumatic brain injury (TBI), accounting for $80 \sim 90 \%$ of the total TBI [1], is typically associated with microstructural integrity loss that persists chronically after the initial injury [2]. The long-term sequelae of mild TBI ( $\mathrm{mTBI}$ ) initiate the risks for neurodegeneration and other age-associated conditions, making it a clinically important topic. Recent large-population retrospective and observational studies showed that chronic mTBI associates with a higher risk of Parkinson's disease (hazard ratio: 1.56) and dementia 
(hazard ratio: 1.17) [3, 4]. However, neuroimaging biomarkers that evaluate the individual-based high risks of age-associated or neurodegenerative deterioration in the brain for mTBI patients are still scarce.

An increasing number of researches have demonstrated that MRI metrics strongly relate to chronological age $[5,6]$. The deviation between chronological age and predicted brain age can reflect how brain diseases interact with normal aging [7-10] and predict the risk of neurodegenerative diseases [11]. The increases in brain-predicted age difference (brain-PAD = predicted brain age - chronological age) have been widely presented in psychiatric disorders and Alzheimer's disease [12, 13]. Recently, Cole et al. observed a 4.66-year increase in brain-PAD estimated by morphometrics in white matter and a 5.97-year increase estimated by gray matter morphometrics in chronic moderate/severe TBI patients instead of the mild cases [10]. However, these results limited our understandings of how mTBI accelerated the brainaging process because only 17 patients with $\mathrm{mTBI}$ were included in their research. The majority of TBIs sustained in civilian contexts are within the mild range [14], which is associated with increased risks of dementia in older adults [15]. The diffuse axonal injury lead by $\mathrm{MTBI}$ [16] causes the long-term deterioration in white matter tracts (WMTs) integrity [17], which is mainly responsible for remote neurodegeneration and aging-related conditions $[18,19]$. Therefore, we hypothesized that MTBI might accelerate the aging process by loss of microstructural integrity.

The relevance vector regression (RVR) [20] was applied to build a brain-age prediction model in 523 healthy individuals. This model was then used to estimate brain age in 116 acute mTBI patients and 63 healthy controls (HCs). Fifty patients were followed up $6 \sim 12$ months to evaluate the longitudinal changes in brain-PAD. Another independent 70 patients were included as a replicated cohort. We investigated whether brain-PAD was prone to be greater in patients with elderly age, post-concussion complaints, and risky apolipoprotein E (APOE) genotype [15, 21-23], and whether it can predict the neuropsychological outcome for information processing speed (IPS). In addition, we hypothesized that white matter tracts contributing to the increased brain age in $\mathrm{MTBI}$ patients were primarily located in the aging-degenerative regions.

\section{Materials And Methods}

\section{Participants}

Training set. Diffusion-tensor imaging (DTI) scans from 523 anonymized healthy individuals were used to build the brain-age prediction model (details in eMethods of Supplemental Materials and Supplemental Table e1).

Test sets. The The flow of participants through the study is shown in Figue 1. There were 116 acute mTBI patients recruited from the local emergency department between 2016 and 2018. Sixty-four patients consented to join in the follow-up investigations, and 50 of them were finally followed 6 12 month postinjury. Screening for mTBI was based on the World Health Organization's Collaborating Centre for Neurotrauma Task Force [24] (details in Table 1). The retrospective baseline health conditions in patients (i.e., hyperglycaemia, hypertension, and hyperlipidemia) were recorded at their first visit. Healthy controls 
( $\mathrm{HCs}, \mathrm{N}=63$ ) were enrolled through the advisements and carefully screened for any neurological or psychiatric disorders. In addition, an independent cohort including 70 acute mTBls with different MRI scanner was included for replication use (details in eMethods of Supplemental Materials). All the subjects gave written, informed consent in person approved by the local institutional review board and conducted in accordance with the Declaration of Helsinki.

\section{Clinical Symptom and Neuropsychological Assessment for Patients}

Clinical symptoms were assessed at the first visit of patients. The post-concussive symptoms (PCS) was evaluated by the International Classification of Diseases, 10th edition clinical (ICD-10) criteria [25]. Patients with three or more symptoms were classified as with PCS (PCS+) otherwise without (PCS-). According to the anterograde amnesia duration, patients were divided into with anterograde amnesia $(\mathrm{AA}+)$ and without $(\mathrm{AA}-)$.

The information processing speed (IPS) was assessed by the Digit Symbol Coding test [26] and TrailMaking Test (Part A) [27] at both initial and follow-up visits. Z-score composed from these two tests was summarized as the performance of IPS.

\section{APOE Genotyping}

Two-milliliter peripheral blood was collected from each of eighty-one mTBI patients for APOE genotype analysis. DNA was extracted from peripheral blood samples using Omega D3494-01 Blood DNA Midi Kit (Omega Bio-tek, Inc., United States). Two single-nucleotide polymorphisms (SNPs; rs429358 and rs7412) were genotyped to identify APOE genotypes comprised of the APOE- $\varepsilon 2,-\varepsilon 3$, and $-\varepsilon 4$ alleles using Assay Design Suite v2.0 (Agena Bioscience, Inc., San Diego, CA). All genotypes containing the $\varepsilon 4$ allele $(\varepsilon 4 / \varepsilon 4$; $\varepsilon 4 / \varepsilon 3 ; \varepsilon 4 / \varepsilon 2)$ were combined as the composite APOE $\varepsilon 4+$, and others $(\varepsilon 2 / \varepsilon 2 ; \varepsilon 2 / \varepsilon 3 ; \varepsilon 3 / \varepsilon 3)$ were combined as the composite APOE $\varepsilon 4$ -

\section{Neuroimaging Data Acquisition and Preprocessing}

The training dataset was collected from multi-centers, by using different MRI scanners (Table e1 in Supplemental Materials). The intraclass correlation coefficient (ICC) of the homogeneity of datasets was calculated after preprocessing. The index ICC for fractional anisotropy (FA) was 0.84 , showing the "excellent" homogeneity (ICC > 0.8) for multi-center datasets.

The DTI scans for test sets were acquired from a 3T MRI scanner (GE750) by a single-shot, spin echobased and diffusion-weighted echo planar imaging sequence $\left(T R=8000 \mathrm{~ms}, T E=68 \mathrm{~ms}\right.$, flip angle $=90^{\circ}$, thickness $=2 \mathrm{~mm}$, slices $=75, \mathrm{FOV}=256 \mathrm{~mm} \times 256 \mathrm{~mm}$, matrix size $=128 \times 128$, two averages, voxel size $=2 \mathrm{~mm} \times 2 \mathrm{~mm} \times 2 \mathrm{~mm})$. DTI scans $\left(b=1000 \mathrm{~s} / \mathrm{mm}^{2}\right)$ were acquired with 30 diffusion gradient directions and the diffusion imaging without weighting $(b=0)$ repeated five times.

All raw DTI data were quality controlled (details in eMethods of Supplemental Materials) and preprocessed using FSL5.0.9 software package (http://www.fmrib.ox.ac.uk/fsl/index.html) following the 
standard procedure [28]. Fifty WMTs were selected based on rICBM_DTI_81_WMPM_FMRIB58 1mm atlas and the mean FA value in each WMT was calculated (details in eMethods of Supplemental Materials).

\section{Brain Age Prediction Modeling Procedure}

The brain-age prediction was performed by defining a relevance vector regression (RVR) to model the relationship between FA values and chronological age in the training set, using Scikit-learn Python library. AS a sort of probabilistic extended linear model based on Bayesian formulation, RVR is derived from Relevance Vector Machine (RVM). Concretely, in the current study, the predictive regression model for brain age was trained by estimating the relevance vectors and their corresponding weight distributions by using the type-II maximum likelihood algorithm. The relevance vectors were derived from the input data (i.e., FA values), which represented the prototypical examples associated with the regression target instead of solely separating attributes. The training process was visualized as Fig. 2A-2D (details in eMethods of Supplemental Materials). The prediction accuracy was assessed using the Pearson correlation ( $r$ ) between chronological age and predicted brain age, the proportion of the variation $\left(R^{2}\right)$ explained by the trained model, the mean absolute error (MAE), and root mean squared error (RMSE) of the brain-PAD scores.

\section{Statistical Analysis}

All the statistical analyses were conducted in the SPSS (version 21.0; IBM, Armonk, NY). To examine the increased brain age in test sets, we compared the brain-PAD scores with zero using the Wilcoxon Signedrank test. Mann-Whitney $U$ test was used to examine the differences in PAD between the group with baseline health condition and the group without. Between-group comparison of the brain-PAD scores for mTBI samples and HCs was conducted by the Analysis of Covariance (ANCOVA), adjusting for age, gender, and education. The longitudinal comparison analysis was performed on brain-PAD between acute and chronic phase with the linear mixed-effects model (LME) analysis. Between-group comparisons in brain-PAD scores for PCS subgroups (PCS+ vs. PCS-), APOE genotypes (APOE \&4+ vs. APOE \&4-), AA subgroups (AA+ vs. AA-), and chronological age subgroups (youth: $\leq 30$ years, middle-aged: $30 \sim 50$ years, elderly: $\geq 50$ years) were also conducted by the ANCOVA, adjusting for gender, education, and/or age. Spearman rank-order correlation (rho) was conducted to examine the associations of brain-PAD scores with education, post-injury time, and Z-score of IPS performance. For the mTBI cohort, the rho between brain-PAD and FA value of WMTs involved in model training was calculated to evaluate the related WMTs contributing to the increased brain age. Results were considered significant under $\mathrm{P}<.05$ (two-tail). Effect sizes for ANCOVA and LME analysis were quantified using partial $\eta^{2}$; effect sizes for the Wilcoxon Signed-rank test and Mann-Whitney $U$ test were quantified by the rank biserial correlation coefficient $\left(r_{r b}\right)$.

\section{Results}

\section{Demographic and clinical characteristics of mTBI patients and HCs}


Demographic and clinical information for test sets are reported in Table 2. The mTBI cohort included 116 acute patients ( 58 males, aged $39.7 \pm 11.9$ years, education: $7.9 \pm 3.9$ years; first-visit post-injury: $3.5 \pm$ 3.4 days). There were 8 patients had the retrospective baseline health conditions in (i.e., hyperglycaemia, hypertension, and hyperlipidemia). Sixty-four patients consented to join in the follow-up investigations and 50 of them (78.1\%) were finally followed 6-12 month post-injury (216.2 \pm 117.8 days). Sixty-three HCs were enrolled (31 males, aged $36.7 \pm 12.4$ years, education: $10.9 \pm 6.6$ years). Patients showed worse performance on IPS tests than HCs (TMT_A: $W=2124, P<0.001, r_{r b}=0.41$; DSCT: $W=5224.5, P<0.001$, $\left.r_{r b}=0.45\right)$.

\section{Chronological age predicted by FA values}

The RVR model could accurately predict the individual chronological age for both the training set and the $\mathrm{HC}$ test set. For the training set, age was accurately predicted by FA values $\left(r=0.96, R^{2}=0.93\right.$, MAE $=$ $3.74, R M S E=5.03)$. The mean PAD for the training group was $-0.18( \pm 5.03)$ years. For HC test set, brain age was also accurately predicted $\left(r=0.97, R^{2}=0.94, M A E=2.57, R M S E=3.16\right)$, and the mean PAD was $0.12( \pm 3.19)$ years (details in eResults of Supplemental Materials).

\section{MTBI patients had increased brain age}

The predicted brain age significantly correlated with chronological age in both $\mathrm{HCs}\left(R^{2}=0.93, P<0.001\right)$ and mTBIs $\left(R^{2}=0.87, P<0.001\right)$ (Fig. $\left.3 \mathrm{~A}\right)$. MTBI patients had older predicted-brain age than their chronological age in acute phase (PAD $=2.59 \pm 5.97$ years; $\left.W=4854, P<0.001, r_{r b}=0.431\right)$, which was replicated in an independent mTBI cohort (PAD $=3.26 \pm 4.55$ years; $W=2158, P<0.001, r_{r b}=0.737$ ). Acute mTBI patients had higher brain-PAD scores ( $2.59 \pm 5.97$ years) than that of $\mathrm{HCs}(0.12 \pm 3.19$ years) $\left(F_{1,173}=7.23, P=0.008\right.$, partial $\eta^{2}=0.041$; Fig. 3B $)$.

In order to avoid the educational level and baseline health conditions of patients being confounding factors, we performed additional analysis. The educational years in the mTBI cohort were lower than that of HCs $(P<0.001)$. Therefore, we randomly selected a subgroup of mTBI patients $(\mathrm{N}=108)$ and $\mathrm{HCs}(\mathrm{N}=$ $47)$, who were matched with age, gender and education level, and then compared the difference in brainPAD scores between groups. Acute mTBI patients still held higher brain-PAD scores ( $2.68 \pm 5.99$ years) than $\mathrm{HC}$ group $(0.31 \pm 3.27$ years $)\left(F_{1,173}=6.60, P=0.011\right.$, partial $\left.\eta^{2}=0.042\right)$. Additionally, the correlation analysis showed no statistically significant correlation between brain-PAD and years of education in $\mathrm{mTBI}$ patients $(r h o=-0.137, P=0.143$ ). Considering that baseline health conditions might affect the brain-PAD, we examine the differences between the subgroup of mTBI patients with a history of hyperglycaemia, hypertension, or/and hyperlipidemia $(\mathrm{N}=8)$ and a matched group of patients who were randomly selected from whom without $(\mathrm{N}=8)$. The result showed there was no statistical difference $(\mathrm{W}=$ $34, P=0.88)$ in acute brain-PAD between two group patients. Those results showed the educational years and baseline health conditions of patients had no effects on PAD in the present study. Therefore, all 116 patients were included in the following analysis. 
MTBI patients held relatively stable brain-PAD (2.50 \pm 4.54 years) at $6 \sim 12$ month follow-up derived from acute injury. The longitudinal comparison in brain-PAD between two phase showed no statistical differences $\left(F_{1,112}=0.436, P=0.51\right.$; Fig. $\left.3 C\right)$. In addition, brain-PAD scores were not associated with postinjury days in both acute and chronic phases (acute phase: $r h o=-0.045, P=0.634$; chronic phase: $r$ o $=$ $-0.015, P=0.920$ ). Moreover, brain-PAD derived from the acute phase was inversely related to the IPS performance in both acute phase ( $r h o=-0.294, P=0.001$; Fig. 4A) and 6-12 month follow-up ( $r h o=$ $-0.356, P=0.011 ;$ Fig. 4B).

\section{Neurodegeneration-vulnerable tracts associated with brain-PAD scores}

The rho between brain-PAD and FA value of WMTs involved in model training $(N=33)$ was calculated to evaluate the related WMTs contributing to the increased brain age. The FA value in 16 WMTs presented significantly correlated to the brain-PAD scores in the acute phase. They were mainly located in the commissural, association, and projection fibers (rho: $-0.305 \sim-0.473, P<0.05$ after family-wise error (FWE) correction for multiple comparisons) (Table 3), primarily including the corpus callosum, fornix, corona radiate and superior longitudinal fasciculus. In the chronic phase, the FA value in the body of fornix was still significantly related to the brain-PAD at follow-up 6 12 months ( $r h o=-0.434, P<0.05$, after FWE correction for multiple comparisons) (Table 3).

\section{Factors affecting on brain-PAD scores}

The patients' chronological age at mTBI occurring affected their PAD derived from acute phase $\left(F_{2,111}=\right.$ $10.05, P<0.001$, partial $\eta^{2}=0.15$ ), after adjusting for sex and education (Table 4). Brain-PAD scores in the elderly patients $(N=27,6.74 \pm 5.61$ years) were higher than the middle-aged $(N=57,1.98 \pm 5.44$ years) and youth patients ( $N=32,0.18 \pm 5.66$ years) (multiple comparisons for Bonferroni correction, $P<$ $0.05)$ (Fig. 5A). The PCS severity post-injury also affected the PAD in acute phase $\left(F_{1,111}=5.26, P=0.02\right.$, partial $\eta^{2}=0.05$ ), after adjusting for sex, education and age. Patients with PCS complaints (i.e., PCS + ) had higher brain-PAD scores $(N=91,3.21 \pm 5.44$ years) than that of PCS- group $(N=25,0.34 \pm 5.50$ years) (Fig. 5B) (Table 4).

The anterograde amnesia (AA) and APOE genotypes did not affect PAD in acute phase (AA: $F_{1,111}=0.86$, $P=0.35$; APOE: $\left.F_{1,111}=0.46, P=0.49\right)$, after adjusting for sex, education and age. Differences in PAD between of $A A+$ subgroup $(N=15,3.84 \pm 5.57$ years) and $A A$ - subgroup $(N=101,2.41 \pm 5.52$ years) was not statistically significant (Table 4). There were also no significant differences in PAD between APOE $\varepsilon 4+(N=17,3.75 \pm 6.31$ years $)$ and APOE $\varepsilon 4$ - subgroups $(N=64,2.57 \pm 6.24$ years) (Table 4$)$.

\section{Discussion}

Our study revealed that mTBI triggered an accelerated aging process in the brain deviating from its chronological trajectory. The increased brain age was observed in the early acute phase and kept stable within a $6 \sim 12$ month follow-up. The WMTs relevant to the accelerated aging-process were primarily 
located in the connecting aging-related and neurodegeneration-vulnerable regions. Besides, the brain-PAD of patients measured in the acute phase could predict the individual performance of IPS at $6 \sim 12$ month follow-up. Patients with older chronological age and severer PCS complaints were more vulnerable to getting "older" brain age.

Unlike one recent study about increased brain-PAD observed in chronic patients (i.e., averaged over 49month post-injury) [29], the observation in our research covered a relatively early phase of mTBI. The results showed that the discrepancy between predicted brain age and chronological age (i.e., brain-PAD > 2 years) was detectable even within the 7-day post-injury. Moreover, the increased brain age cannot simply be explained as a transient physiological pattern in response to traumatic events. Instead, the current longitudinal observation on brain-PAD further demonstrated such an accelerated brain-aging process was a stable change. As described in neurological and neuropsychiatric researches, the increases in brain age have been considered as a surrogate neurobiomarker of vulnerability to neurodegeneration $[7,30]$. Likewise, the brain-PAD score may be termed as a candidate biomarker for mTBI. No significant correlations between the brain-PAD score and time since injury in both acute and chronic phases were observed, which further supplemented the explanation that such increases in brain age might reflect the vulnerability to the accelerated aging process.

For both acute phase and follow-up, the increases in brain age inversely related to the integrity of fornix. These evidences implied that the deteriorated integrity of fornix is mainly responsible for the persistent brain-aging process accelerated by mTBI. The fornix, connecting the hippocampal formation in the limbic system, is responsible for neurodegeneration progression [31]. Recent theory about the transmission model in Alzheimer's disease (AD) shows that the pattern spreads initially from the transentorhinal cortex and hippocampus latterly to the rest of the brain [32,33]. Moreover, alteration of microstructure organization in the fornix is also responsible for neurodegeneration in mild cognitive impairment and chronological aging process $[34,35]$. Those evidences in neurodegenerative conditions suggests that the fornix may serve as a common connecting origin of neurodegeneration in the transmission pathway. Therefore, based on the current findings on the steady association between the integrity of fornix and increased brain age, we can infer that brain-PAD score deriving from the WMT integrity was informative in evaluating the potential neurodegeneration effects.

We explored factors affecting brain-PAD in patients with $\mathrm{mTBI}$, which could explain the individual differences in the acceleration of brain-aging post-injury. The results showed that patients with older chronological age and severer PCS complaints were more vulnerable to getting greater brain-PAD. These results were consistent with some retrospective studies' findings that post-concussion syndrome and older age increased the risks of developing dementia for patients with $\mathrm{mTBI}[15,21]$. The APOE genotypes or anterograde amnesia in our study showed non-significant effects on brain-PAD following mTBI. Both APOE $\varepsilon 4$ and anterograde amnesia are generally considered as risk factors for $A D[22,23]$. Those provides some possible clues that the neurodegeneration-vulnerable aging process accelerated by $\mathrm{mTBI}$ may be differential from AD pathology [36]. However, there still call for further studies to explore the common and dissociated factors contributing to the neurodegeneration in $\mathrm{mTBI}$ and $\mathrm{AD}$ 
Our model explained the variations in the chronological aging process as aging-related changes in WMTs. Unlike previous studies using macroscopically morphological features to predict brain age in moderate to severe TBI patients [10,29], we adopted more subtle microstructural features, considering mTBI usually causes diffuse axon injury [16] and long-distance WM disconnections [18]. Those damages, thereafter, result in a degradation in the efficiency of communication among brain regions and contribute to cognitive dysfunctions during the brain-aging process, commonly reflected as slow speed of information processing and transferring [37]. Moreover, our results demonstrated that brain-PAD in the acute phase could predict the long-term individual profiles on the information processing speed (IPS). The greater brain-PAD was relevant to poorer performance on IPS, which suggests that WM integrity loss contributed to the cognitive impairments following mTBI. Additionally, the potential of WMTs as predictors for brainPAD post-injury, discussed above, further held the fact that $\mathrm{mTBI}$ might be vulnerable to tracts primarily connecting the neurodegeneration-related regions.

There are limitations to the present study. Firstly, our brain-age prediction model was trained with DTI metrics with respect to injury characteristics of mTBI. The brain-aging process is biologically complex, which may be explained more by the multi-modality of neuroimaging. Secondly, in the current longitudinal study, we observed that the brain-PAD (i.e., 2.59 years) was stable within one-year, which reflected the aging-related changes persistently existing in white matter tracts after the injury. It still needs to be careful to infer whether or not this acceleration in brain aging process is responsible for the neurodegeneration, because the neurodegeneration following injury may contain a chronic interaction of neuroinflammation with damages to WMTs integrity [38]. Besides, the follow-up loss and insufficient APOE $\varepsilon 4$ samples might also limit our understandings of the association between the accelerated brainaging process following $\mathrm{mTBI}$ and neurodegenerative diseases, such as AD.

\section{Conclusions}

In summary, the acceleration of the brain-aging process following $\mathrm{mTBI}$ is detectable even in the early acute phase and persists into the chronic phase, which provides insights into the risks of mTBI for neurodegeneration-vulnerable conditions. Moreover, the increases in brain age are related to clinical settings and capable of predicting neuropsychological outcomes.

\section{Abbreviations}

$\mathrm{PAD}=$ predicted age difference; $\mathrm{mTBI}=$ mild traumatic brain injury; $\mathrm{FA}$ = fractional anisotropy; $\mathrm{WMT}=$ white matter tract; RVR = relevance vector regression; $D T I$ = diffusion-tensor imaging; IPS = information processing speed; $\mathrm{PCS}$ = post-concussive symptom; $\mathrm{APOE}$ = apolipoprotein $\mathrm{E}$.

\section{Declarations}

Ethics approval and consent to participate 
Patient consent was Obtained. Ethics approval The Second Affiliated Hospital of Wenzhou Medical University.

\section{Consent for publication}

All the authors have approved the manuscript.

\section{Availability of data and materials}

All the data mentioned in this article are available on published article.

\section{Competing interests}

The authors have no conflicts of interest to declare.

\section{Funding}

Study Funded by the National Natural Science Foundation of China [Grant Nos. 81771914, 81871331, 81671671], Fundamental Research Funds for the Central Universities [Grant Nos. Xjj 2018229, xzy022019045], Wenzhou Science and Technology Bureau in China [Grant No. Y20180112], Changsha Science and technology project [Grant No. kq1801115].

\section{Authors' Contributions}

Shuoqiu Gan, Ming Zhang, and Lijun Bai participated in the design of the study. Shuoqiu Gan and Wen Shi drafted the manuscript. Bo Yin, Guanghui Bai, and Zhuonan Wang were involved in data collection. Yingxiang Sun carried out the APOE analysis. Shan Wang, Xiaoyan Jia, and Xuan Niu analyzed the data. Wen Shi, Shuoqiu Gan, and Chuanzhu Sun participated in the RVR model training. Xiaofan Jiang, Jun Liu and Lijun Bai revised the manuscript. All authors read and approved the final manuscript.

\section{Acknowledgement}

We thank all patients and healthy volunteers that participated in the study. We also give sincere gratitude to the data sharing from IXI (http://brain-development.org/ixi-dataset/), CoRR (http://fcon_1000.projects.nitrc.org/indi/CoRR/html/), and Cyclotron Research Centre (University of Liège) (https://www.nitrc.org/projects/parktdi/).

\section{References}

1. Levin HS, Diaz-Arrastia RR: Diagnosis, prognosis, and clinical management of mild traumatic brain injury. The Lancet Neurology 2015, 14(5):506-517.

2. Mac Donald CL, Barber J, Andre J, Panks C, Zalewski K, Temkin N: Longitudinal neuroimaging following combat concussion: sub-acute, 1 year and 5 years post-injury. Brain communications 2019, 1(1):fcz031. 
3. Gardner RC, Byers AL, Barnes DE, Li Y, Boscardin J, Yaffe K: Mild TBI and risk of Parkinson disease: A Chronic Effects of Neurotrauma Consortium Study. Neurology 2018:10.1212/WNL. 0000000000005522.

4. Fann JR, Ribe AR, Pedersen HS, Fenger-Grøn M, Christensen J, Benros ME, Vestergaard M: Long-term risk of dementia among people with traumatic brain injury in Denmark: a population-based observational cohort study. The Lancet Psychiatry 2018, 5(5):424-431.

5. Cox SR, Ritchie SJ, Tucker-Drob EM, Liewald DC, Hagenaars SP, Davies G, Wardlaw JM, Gale CR, Bastin ME, Deary IJ: Ageing and brain white matter structure in 3,513 UK Biobank participants. Nature communications 2016, 7:13629.

6. Good CD, Johnsrude IS, Ashburner J, Henson RN, Friston KJ, Frackowiak RS: A voxel-based morphometric study of ageing in 465 normal adult human brains. Neurolmage 2001, 14(1):21-36.

7. Wang J, Knol MJ, Tiulpin A, Dubost F, de Bruijne M, Vernooij MW, Adams HH, Ikram MA, Niessen WJ, Roshchupkin GV: Gray Matter Age Prediction as a Biomarker for Risk of Dementia. Proceedings of the National Academy of Sciences 2019:201902376.

8. Schnack HG, Van Haren NE, Nieuwenhuis M, Hulshoff Pol HE, Cahn W, Kahn RS: Accelerated brain aging in schizophrenia: a longitudinal pattern recognition study. American Journal of Psychiatry 2016, 173(6):607-616.

9. Cole JH, Annus T, Wilson LR, Remtulla R, Hong YT, Fryer TD, Acosta-Cabronero J, Cardenas-Blanco A, Smith $\mathrm{R}$, Menon DK: Brain-predicted age in Down syndrome is associated with beta amyloid deposition and cognitive decline. Neurobiology of aging 2017, 56:41-49.

10. Cole JH, Leech R, Sharp DJ, Initiative AsDN: Prediction of brain age suggests accelerated atrophy after traumatic brain injury. Annals of neurology 2015, 77(4):571-581.

11. Cole $\mathrm{JH}$, Franke $\mathrm{K}$ : Predicting age using neuroimaging: innovative brain ageing biomarkers. Trends in neurosciences 2017, 40(12):681-690.

12. Gaser C, Franke K, Klöppel S, Koutsouleris N, Sauer H, Initiative AsDN: BrainAGE in mild cognitive impaired patients: predicting the conversion to Alzheimer's disease. PloS one 2013, 8(6).

13. Koutsouleris N, Davatzikos C, Borgwardt S, Gaser C, Bottlender R, Frodl T, Falkai P, Riecher-Rössler A, Möller H-J, Reiser M: Accelerated brain aging in schizophrenia and beyond: a neuroanatomical marker of psychiatric disorders. Schizophrenia bulletin 2014, 40(5):1140-1153.

14. Cassidy JD, Carroll L, Peloso P, Borg J, Von Holst H, Holm L, Kraus J, Coronado V: Incidence, risk factors and prevention of mild traumatic brain injury: results of the WHO Collaborating Centre Task Force on Mild Traumatic Brain Injury. Journal of rehabilitation medicine 2004, 36(0):28-60.

15. Gardner RC, Burke JF, Nettiksimmons J, Kaup A, Barnes DE, Yaffe K: Dementia risk after traumatic brain injury vs nonbrain trauma: the role of age and severity. JAMA neurology 2014, 71(12):14901497.

16. Smith DH, Meaney DF, Shull WH: Diffuse axonal injury in head trauma. The Journal of head trauma rehabilitation 2003, 18(4):307-316. 
17. Mac Donald CL, Johnson AM, Cooper D, Nelson EC, Werner NJ, Shimony JS, Snyder AZ, Raichle ME, Witherow JR, Fang R: Detection of blast-related traumatic brain injury in US military personnel. New England journal of medicine 2011, 364(22):2091-2100.

18. Sharp DJ, Scott G, Leech R: Network dysfunction after traumatic brain injury. Nature Reviews Neurology 2014, 10(3):156.

19. Smith DH, Johnson VE, Stewart W: Chronic neuropathologies of single and repetitive TBI: substrates of dementia? Nature Reviews Neurology 2013, 9(4):211.

20. Tipping ME: Sparse Bayesian learning and the relevance vector machine. Journal of machine learning research 2001, 1(Jun):211-244.

21. Carroll L, Cassidy JD, Peloso P, Borg J, Von Holst H, Holm L, Paniak C, Pépin M: Prognosis for mild traumatic brain injury: results of the WHO Collaborating Centre Task Force on Mild Traumatic Brain Injury. Journal of rehabilitation medicine 2004, 36(0):84-105.

22. Jagust W: Vulnerable neural systems and the borderland of brain aging and neurodegeneration. Neuron 2013, 77(2):219-234.

23. Nicoll JA, Roberts GW, Graham DI: Apolipoprotein E $\varepsilon 4$ allele is associated with deposition of amyloid $\beta$-protein following head injury. Nature medicine 1995, 1(2):135.

24. Holm L, David Cassidy J, Carroll L, Borg J: Summary of the WHO collaborating centre for neurotrauma task force on mild traumatic brain injury. Journal of rehabilitation medicine 2005 , 37(3):137-141.

25. Organization WH: The ICD-10 classification of mental and behavioural disorders: clinical descriptions and diagnostic guidelines: Geneva: World Health Organization; 1992.

26. Ryan JJ, Ward LC: Validity, reliability, and standard errors of measurement for two seven-subtest short forms of the Wechsler Adult Intelligence Scale-III. Psychological Assessment 1999, 11(2):207.

27. Bowie CR, Harvey PD: Administration and interpretation of the Trail Making Test. Nature protocols 2006, 1(5):2277.

28. Jenkinson M, Beckmann CF, Behrens TEJ, Woolrich MW, Smith SM: FSL. Neurolmage 2012, 62(2):782-790.

29. Savjani RR, Taylor BA, Acion L, Wilde EA, Jorge RE: Accelerated changes in cortical thickness measurements with age in military service members with traumatic brain injury. Journal of neurotrauma 2017, 34(22):3107-3116.

30. Cole JH, Franke K: Predicting age using neuroimaging: innovative brain ageing biomarkers. Trends in neurosciences 2017.

31. D'Esposito M, Verfaellie M, Alexander MP, Katz DI: Amnesia following traumatic bilateral fornix transection. Neurology 1995, 45(8):1546-1550.

32. Ahmed Z, Cooper J, Murray TK, Garn K, McNaughton E, Clarke H, Parhizkar S, Ward MA, Cavallini A, Jackson S: A novel in vivo model of tau propagation with rapid and progressive neurofibrillary tangle 
pathology: the pattern of spread is determined by connectivity, not proximity. Acta neuropathologica 2014, 127(5):667-683.

33. Iba M, Guo JL, McBride JD, Zhang B, Trojanowski JQ, Lee VM-Y: Synthetic tau fibrils mediate transmission of neurofibrillary tangles in a transgenic mouse model of Alzheimer's-like tauopathy. Journal of Neuroscience 2013, 33(3):1024-1037.

34. Jacquemont T, Fallani FDV, Bertrand A, Epelbaum S, Routier A, Dubois B, Hampel H, Durrleman S, Colliot $\mathrm{O}$, Initiative AsDN: Amyloidosis and neurodegeneration result in distinct structural connectivity patterns in mild cognitive impairment. Neurobiology of aging 2017, 55:177-189.

35. de Groot M, Cremers LG, Ikram MA, Hofman A, Krestin GP, van der Lugt A, Niessen WJ, Vernooij MW: White matter degeneration with aging: Iongitudinal diffusion MR imaging analysis. Radiology 2015, 279(2):532-541.

36. Weiner MW, Harvey D, Hayes J, Landau SM, Aisen PS, Petersen RC, Tosun D, Veitch DP, Jack Jr CR, Decarli C: Effects of traumatic brain injury and posttraumatic stress disorder on development of Alzheimer's disease in Vietnam veterans using the Alzheimer's Disease Neuroimaging Initiative: preliminary report. Alzheimer's \& Dementia: Translational Research \& Clinical Interventions 2017, 3(2):177-188.

37. O'Sullivan M, Jones DK, Summers P, Morris R, Williams S, Markus H: Evidence for cortical "disconnection" as a mechanism of age-related cognitive decline. Neurology 2001, 57(4):632-638. 38. Armstrong RC, Mierzwa AJ, Marion CM, Sullivan GM: White matter involvement after TBI: Clues to axon and myelin repair capacity. Experimental neurology 2016, 275:328-333.

\section{Tables}


Table 1

The inclusion and exclusion criteria for mTBI

\section{Inclusion and Items}

exclusion criteria

Inclusion criteria i). Glasgow Coma Score of 13-15

ii). One or more of the following:

a. Loss of consciousness (if present) $<30 \mathrm{~min}$;

b. Post-traumatic amnesia (if present) $<24 \mathrm{~h}$;

c. Other transient neurological abnormalities such as focal signs and seizure

iii). No contraindications to MRI

Exclusion criteria

i). History of neurological disease

ii). History of psychiatric disorder

ii). History of head injury

邓). History of substance or alcohol abuse

邓). History of intubation and/or presence of a skull fracture and administration of sedatives on arrival in the emergency department

邓). Spinal cord injury

邓). Manifestation of mTBI due to other reasons:

a. Due to medications by other injuries (e.g., systemic injuries, facial injuries, or intubation);

b. Caused by other problems (e.g., psychological trauma, language barrier, or coexisting medical conditions)

c. Caused by penetrating cranio-cerebral injury

Note $-\min =$ Minites; $\mathrm{h}=$ Hours 
Table 2

Demographic and clinical characteristics of mTBI cohort and healthy controls

\begin{tabular}{|c|c|c|c|}
\hline Demographic and Clinical Characteristics & $\begin{array}{l}\text { mTBI Cohort } \\
(\mathrm{N}=116)\end{array}$ & $\begin{array}{l}\text { HCs } \\
(N=63)\end{array}$ & $P$ value \\
\hline Age (Years) & $39.7 \pm 11.9$ & $36.7 \pm 12.4$ & 0.101 \\
\hline Sex (Male, female) & 58,58 & 31,32 & 0.919 \\
\hline Education (Years) & $7.9 \pm 3.9$ & $10.9 \pm 6.6$ & $0.001^{*}$ \\
\hline First-visit post-injury (Days) & $3.5 \pm 3.4$ & - & - \\
\hline \multicolumn{4}{|l|}{ Injury cause } \\
\hline Traffic accident (\%) & 57.8 & - & - \\
\hline Fall (\%) & 20.7 & - & - \\
\hline Assault (\%) & 11.2 & - & - \\
\hline Other $(\%)$ & 10.3 & - & - \\
\hline \multicolumn{4}{|l|}{ Injury severity } \\
\hline $\operatorname{PCS}(+/-)$ & $91 / 25$ & - & - \\
\hline Coma duration (Minutes) & $11.3 \pm 10.8$ & - & - \\
\hline AA duration (Hours) & $0.4 \pm 1.7$ & - & - \\
\hline IPS & $31.3 \pm 15.9$ & $45.7 \pm 18.1$ & $<0.001^{*}$ \\
\hline DSCT & $72.3 \pm 49.8$ & $44.8 \pm 30.9$ & $<0.001^{*}$ \\
\hline \multicolumn{4}{|l|}{ TMT_A (Seconds) } \\
\hline \multicolumn{4}{|c|}{$\begin{array}{l}\text { Note.- P values were derived from with the } \chi^{2} \text { test or Mann-Whitney U test, as appropriate. } \\
\text { Abbreviation: HCS = Healthy Controls; PCS + = With Post Concussion Symptom complaints; PCS - = } \\
\text { Without Post Concussion Symptom complaints; AA duration = Anterograde amnesia duration; IPS = } \\
\text { Information processing speed; DSCT = Digit Symbol coding test; TMT_A = Trail-Making Test Part A }\end{array}$} \\
\hline
\end{tabular}


Table 3

Correlation between FA value of age-sensitive WMTs and brain-PAD in acute and chronic phase

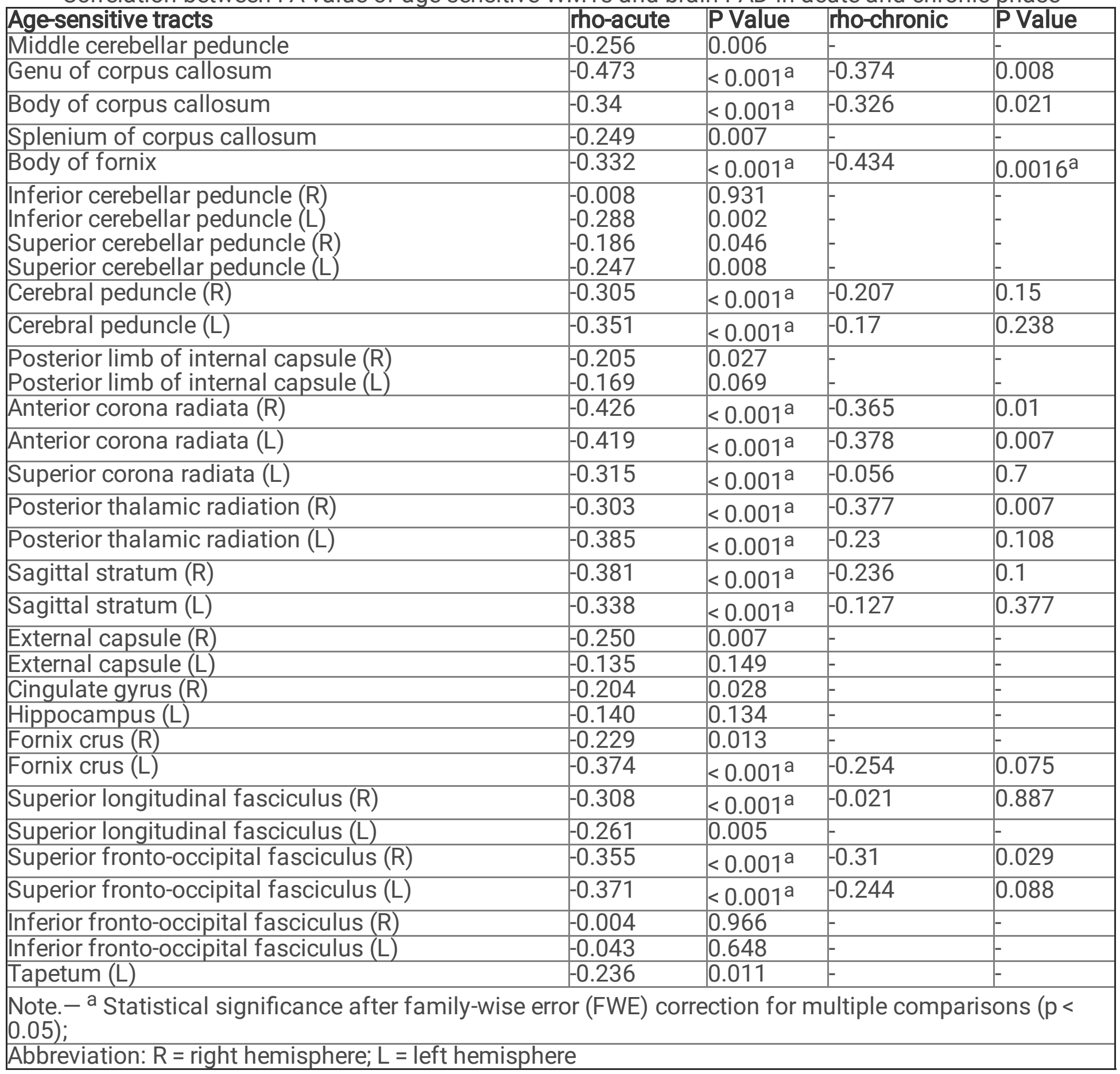


Table 4

Individual differences in brain-PAD scores post-injury

\begin{tabular}{|c|c|c|c|c|}
\hline & Brain-PAD (Years) & F value & P value & partial $\eta^{2}$ \\
\hline Chronological age & & \multirow[t]{4}{*}{10.05} & \multirow[t]{4}{*}{$<0.001^{a}$} & \multirow[t]{4}{*}{0.15} \\
\hline Youth $(\mathrm{N}=32)$ & $0.18 \pm 5.66$ & & & \\
\hline Adults $(\mathrm{N}=57)$ & $1.98 \pm 5.44$ & & & \\
\hline Elderly $(\mathrm{N}=27)$ & $6.74 \pm 5.61$ & & & \\
\hline PCS & & \multirow[t]{3}{*}{5.26} & \multirow{3}{*}{$0.02^{a}$} & \multirow[t]{3}{*}{0.05} \\
\hline $\mathrm{PCS}+(\mathrm{N}=91)$ & $3.21 \pm 5.44$ & & & \\
\hline PCS- $(\mathrm{N}=25)$ & $0.34 \pm 5.50$ & & & \\
\hline AA & & \multirow[t]{3}{*}{0.86} & \multirow[t]{3}{*}{0.35} & \\
\hline $\mathrm{AA}+(\mathrm{N}=15)$ & $3.84 \pm 5.57$ & & & \\
\hline $\mathrm{AA}-(\mathrm{N}=101)$ & $2.41 \pm 5.52$ & & & \\
\hline $\mathrm{APOE}$ & & \multirow[t]{3}{*}{0.46} & \multirow[t]{3}{*}{0.49} & \\
\hline$\varepsilon 4+(N=17)$ & $3.75 \pm 6.31$ & & & \\
\hline$\varepsilon 4-(\mathrm{N}=64)$ & $2.57 \pm 6.24$ & & & \\
\hline
\end{tabular}

Figures

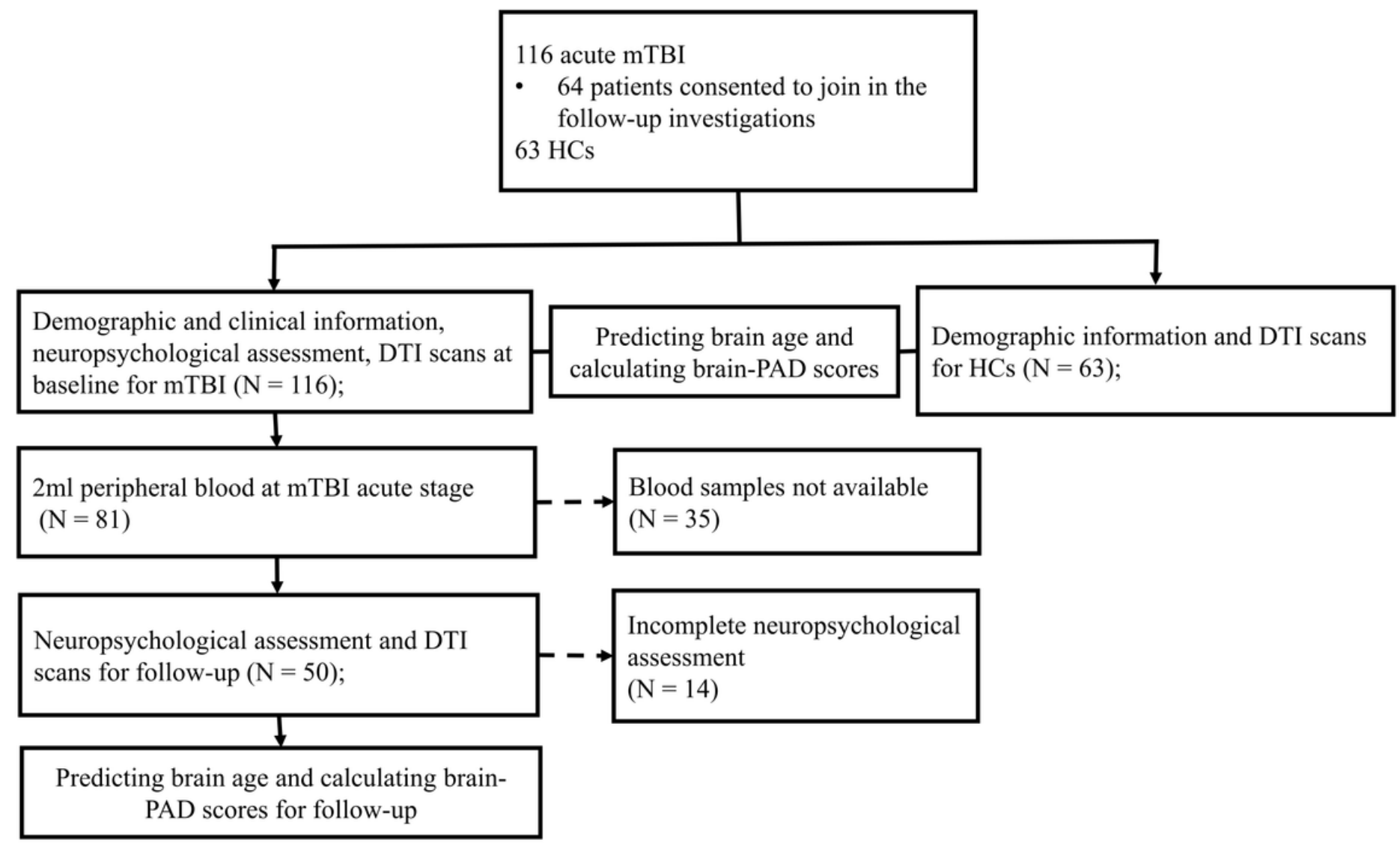

Figure 1

The flow diagram shows the inclusion process for participants of test sets. Abbreviations: brain-PAD = brain-predicted age difference; $\mathrm{mTBI}=$ mild traumatic brain injury; $\mathrm{HCs}=$ healthy controls. 
> Training set:

Healthy participants,

$\left(\mathrm{N}_{\mathrm{tr}}=523,11 \leq\right.$ Age $\left.\leq 86\right)$
Test set: HCs

Healthy controls

$\left(\mathrm{N}_{\mathrm{te}}=63,20 \leq\right.$ Age $\left.\leq 63\right)$
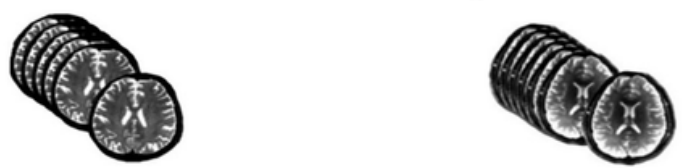

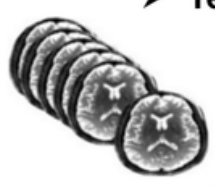

a. Acute phase

$(\mathrm{N}=116,20 \leq \mathrm{Age} \leq 64)$
Follow-up

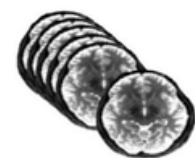

b. Chronic phase

$(\mathrm{N}=50,21 \leq$ Age $\leq 60)$

All DTI image preprocessing in FSL, and extracting mean FA value in each tract

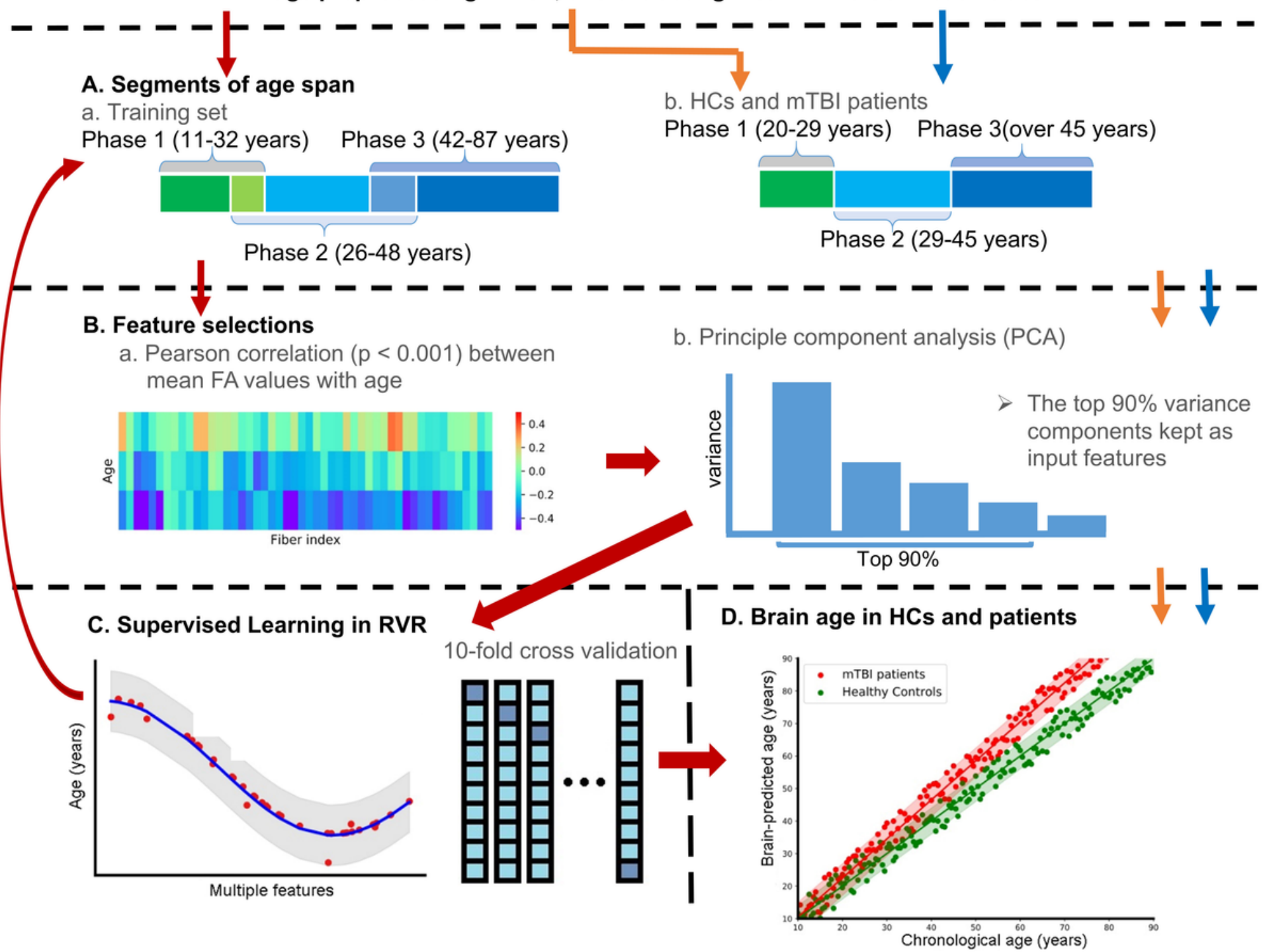

Figure 2

Overview of brain age prediction model. (A) The model was performed in a given segment of the age span. (a) In the training set, the age span (11 87 years) was partitioned into three segments (phase 1: 11 $\sim 32$ years, $N=186$; phase 2: $26 \sim 48$ years, $N=156$; phase 3: $42 \sim 87$ years, $N=272$ ). Two adjacent age phases in the training set were slightly overlapped (i.e., 6-year overlap) to enhance the tolerance towards the inter-individual variability of brain age prediction. (b) In the test sets, each age span segment corresponded with the age phase in the training set, in which the overlapped part of two adjacent phases was averaged (phase 1: $20 \sim 29$ years; phase 2: $29 \sim 45$ years; phase 3: over 45 years). (B) Feature selections contained two steps: (a) Pearson correlation coefficient $(r)$ was calculated $(P<0.001)$ between 
the mean FA value of each WMT and age in each age segment of the training set, and WMT with the absolute value of $r$ above 0.25 was kept; (b) Dimensionality of features was then reduced by Principle Components Analysis (PCA). The components with the top $90 \%$ variance were kept as the input features.

(C) The supervised learning process was conducted using Relevance vector regression (RVR) to define the relationship between the chronological ages and input features. Internal validation was assessed by running 10-fold cross-validation on the shuffled training set with the same error calculation of the test set. (D) The trained RVR brain-age model was entirely applied to all test sets.
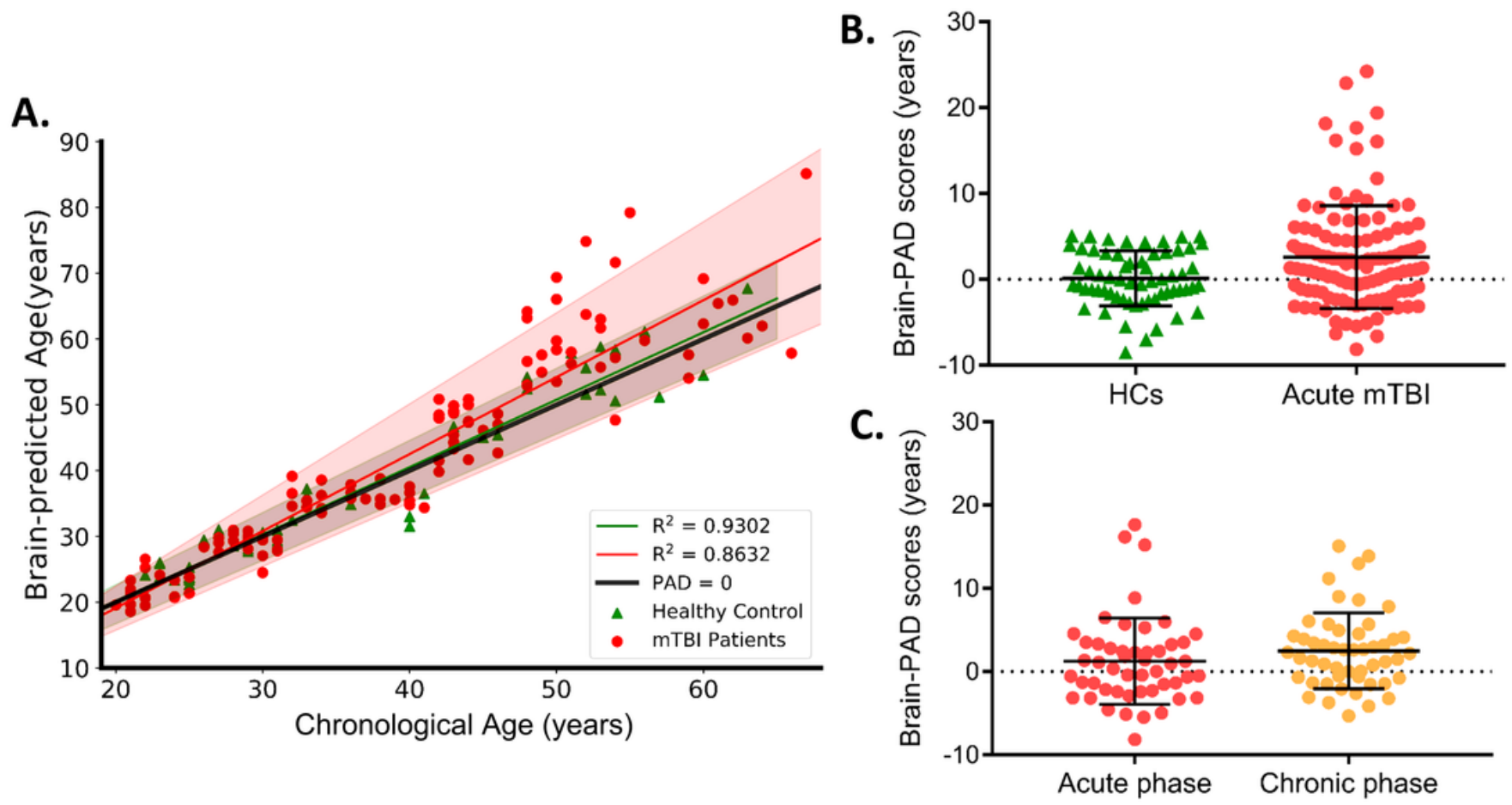

\section{Figure 3}

MTBI increased the brain-aging process. (A) Scatterplot and linear regression of individual chronological age and brain-predicted age in each test set derived from the RVR prediction model. Points and triangles indicated $\mathrm{mTBI}$ individuals in the acute phase and HCs respectively, and lines were regression lines for each group $(\mathrm{mTBI}=$ red; healthy control = green), with 95\% confidence interval displayed. Both two regression lines of chronological age and brain-predicted age were fitted well (HCs: $R 2=0.94, P<0.001$; acute $\mathrm{mTBI}: \mathrm{R} 2=0.87, \mathrm{P}<0.001)$. The solid black line (black) was the line of identity $(y=x)$. (B) The cross-sectional comparison (with error bar) of brain-PAD scores between acute mTBI patients and HCs. The brain-PAD scores in acute $\mathrm{mTBI}$ individuals ( $2.59 \pm 5.97$ years) were significantly higher than both zero $(P<0.001)$ and the brain-PAD scores in HCs $(0.12 \pm 3.19$ years, $P=0.008)$. (C) The longitudinal comparison of brain-PAD scores between the acute and chronic phases in patients with mTBI. The brainPAD scores in mTBI patients were still significantly higher than zero in their chronic phase $(2.50 \pm 4.54$ years, $P<0.001)$, but not significantly higher than scores in the acute phase $(1.87 \pm 5.66$ years, $P=0.51)$. Abbreviations: brain-PAD = brain-predicted age difference; $\mathrm{HC}=$ healthy control; $\mathrm{mTBI}=$ mild traumatic brain injury. 


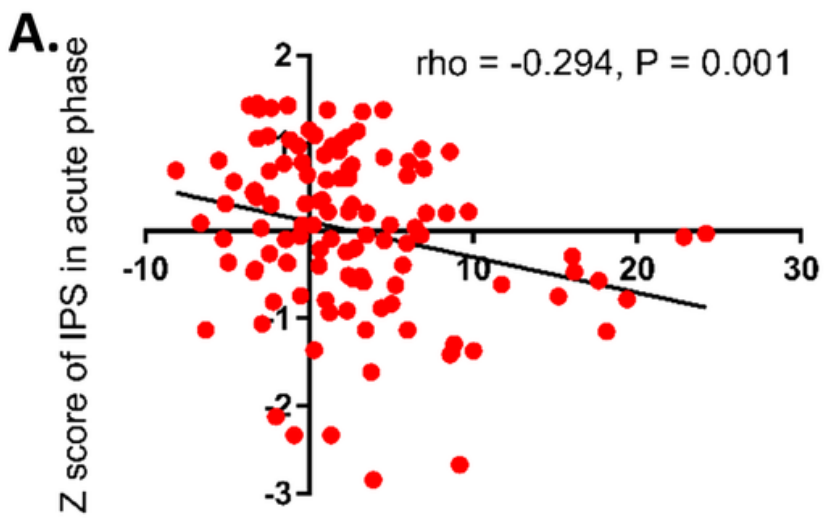

PAD in acute phase (years)

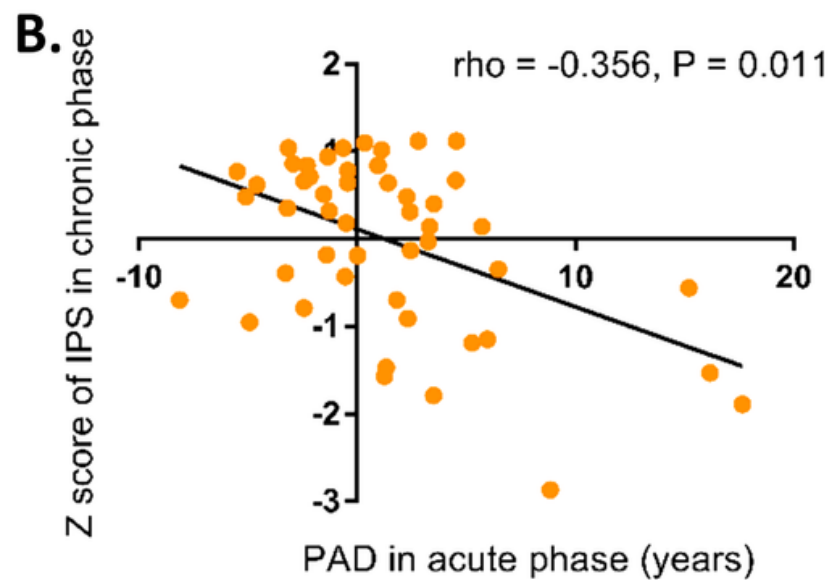

\section{Figure 4}

PAD in the acute phase predicted neuropsychological outcomes. (A) In the acute phase, the increased brain-PAD related to the decreased IPS $(P=0.001)$. (B) Patients with greater PAD in the acute phase still performed slower on the IPS in the chronic phase. $(P=0.011)$. Abbreviations: IPS = information processing speed; $P A D=$ predicted age difference; rho = Spearman rank-order correlation coefficient.
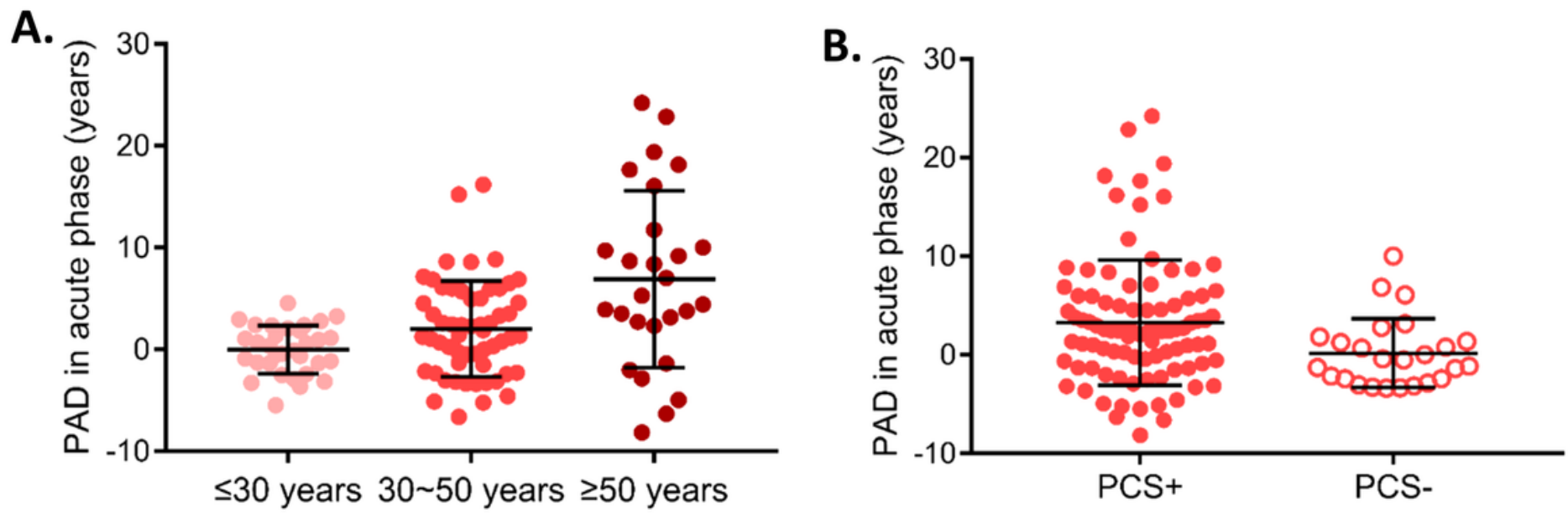

\section{Figure 5}

Individual differences in PAD. (A) Patients' chronological age at mTBI occurring affected their PAD in the acute phase. The elderly patients ( $\geq 50$ years) had greater PAD (6.74 \pm 1.08 years) than the middle-aged (30 50 years) (1.98 \pm 0.72 years) and the youth ( $\leq 30$ years) $(0.18 \pm 1.00$ years). (B) The PCS severity post-injury affected the PAD in the acute phase. Patients with severer complaints on PCS had greater PAD (3.262 \pm 6.350 years) than those without PCS complaints $(0.159 \pm 3.488$ years). Abbreviations: PCS $+=$ with post-concussion symptom complaints; $\mathrm{PCS}-=$ without post-concussion symptom complaints; $\mathrm{PAD}=$ predicted age difference.

\section{Supplementary Files}


This is a list of supplementary files associated with this preprint. Click to download.

- SupplementalTablee1.docx

- SupplementalMaterials.docx 